\title{
Efficient multi sub-band Monte Carlo simulation of nano-scaled Double Gate MOSFETs
}

\author{
Jérôme Saint-Martin, Damien Querlioz, Arnaud Bournel and Philippe Dollfus \\ Institut d'Electronique Fondamentale, CNRS UMR 8622 \\ Bât. 220, Université Paris Sud \\ F-91405 Orsay cedex France \\ E-mail: jerome.saint-martin@ief.u-psud.fr
}

\begin{abstract}
An efficient two-dimensional self-consistent MonteCarlo simulator including multi sub-band transport in a 2D electron gas is described. This simulator takes into account both out of equilibrium transport and quantization effects. A new algorithm improves significantly computation time and allows us to study the sub-threshold behavior of deep sub-100 nm CMOS devices. We analyze quantization effects in a $15 \mathrm{~nm}$-long DGMOS transistor.
\end{abstract}

Keywords- MOSFET, Multi sub-band, Monte Carlo, Double gate, Ballistic transport, $2 D$ Electron Gaz.

\section{INTRODUCTION}

Double gate (DG) structure are promising architectures to overcome short channel effects in nanometer scaled MOSFETs. To obtain good performance in both the off and on states, DG scaling requires ultra-thin body thickness $\mathrm{T}_{\mathrm{Si}}$, typically less than $10 \mathrm{~nm}$ for sub $50 \mathrm{~nm}$ MOSFETs [1]. Consequently, quantum confinement in the direction perpendicular to the gate stacks may affect the operation of these devices. Moreover, in ultra-short devices the quasiballistic transport strongly affect device performance [2]. In this context, Monte Carlo (MC) simulation with multi sub-band description is required to properly describe both the quantum vertical confinement in the channel together with accurate scattering and out-of equilibrium effects. However this kind of simulation induces a dramatic increase of computation time, in comparison with traditional semi-classical MC simulation.

We present a new approach for reasonably fast and stable simulation of these devices without loss of accuracy, using a perturbative approach to solving the Schrödinger equation. In particular, this approach makes possible to investigate the subthreshold behavior of transistors within an acceptable computation time.

The first part of the paper is devoted to the presentation of our original multi-sub-band algorithm. Then, we present the acceleration technique. In the last part we analyze the performance of a typical double gate structure, focusing on the sub threshold behavior.

\section{REFERENCE ALGORITHM}

Our model is inspired by the mode-space approach of quantum transport [3] and extends the technique developed in ref.[4]. It is self consistently coupled with 1D Schrödinger's equation and 2D Poisson's equation (cf. Fig 1 and Fig 2).

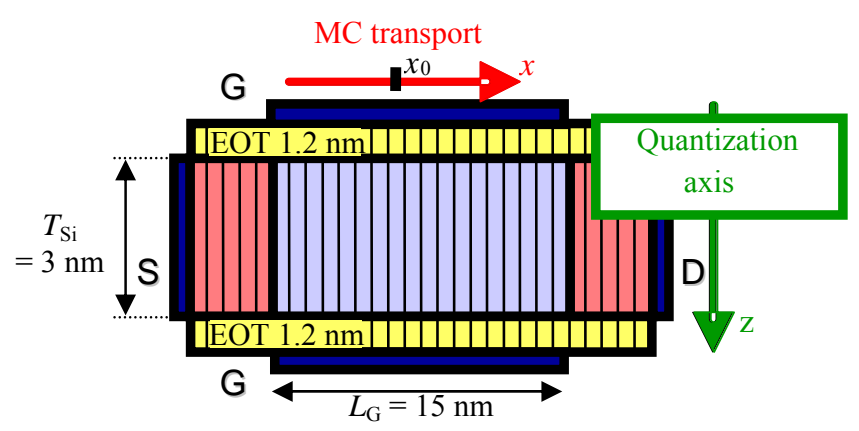

Figure 1. Schematic of DGMOS structure.

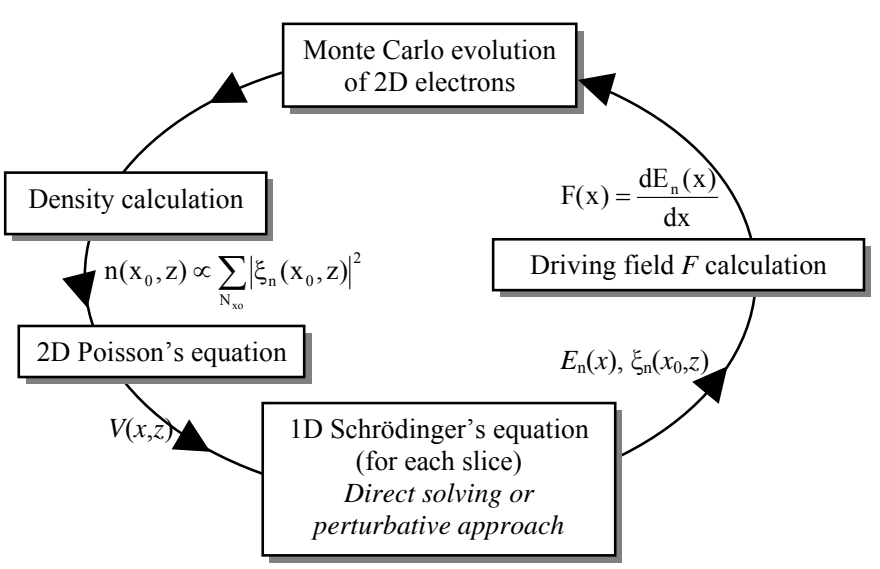

Figure 2. Multi sub-band Monte-Carlo algorithm.

Each time step begins with the solution of the 1D Schrödinger equation along the $z$-axis perpendicular to gate stacks in each $x_{0}$-slice of the DG. This provides the evolution along the source-drain $x$-axis of the eigen energies $E_{\mathrm{n}}\left(x_{0}\right)$ and of the wavefunctions $\xi_{\mathrm{n}}\left(x_{0}, z\right)$ associated with each sub-band numbered ' $n$ '.

Along the Source/Drain $x$-axis, the confined carrier movement is supposed semi classical, and the Boltzmann transport equation is solved using a $\mathrm{MC}$ technique. According to the mode-space approach, the driving field undergone by a

This work was supported by the European SINANO Network of Excellence and by French RMNT and ANR under CMOS-D-ALI and MODERN Projects. 
carrier belonging to sub-band ' $n$ ' is given by the $x$-derivative of the associated energy $E_{\mathrm{n}}$.

Scattering mechanisms included in the MC transport simulation are bulk phonons, roughness scattering and impurity scattering, taking non parabolic and ellipsoidal band structures into account. The acoustic and intervalley phonon scattering rate calculations are detailed in Ref. [5]. The considered phonons and related coupling constants used for the multi subband, or $2 \mathrm{Dk}$, calculations are the same than that considered in semi-classical, or $3 \mathrm{Dk}$, simulation. The surface roughness effect is calculated by considering both influences of electrostatic potential along $z$ [6] and of eigen energies fluctuations [7]. The 2D scattering rates are updated regularly according to the new wave-functions resulting from the Schrödinger equation.

This transport algorithm provides the number of electrons present in each sub-band of the "Schrödinger" $x_{0}$-slice at the end of the time step. The electron density is calculated by distributing the charge of each electron along its $x_{0}$-slice according to the appropriate probability density $\left|\xi_{\mathrm{n}}\left(x_{0}, z\right)\right|^{2}$.

Finally, the 2D Poisson equation is solved by using the electron density $n(x, z)$ derived from the sum of the charge contribution of each particle present in a $x_{0}$-slice of the device

The previous multi sub-band simulator makes possible detailed investigation of vertical confinement in nanoscale devices. It is however about 50 times slower than the corresponding classical simulations.

\section{EFFICIENT ALGORITHM}

In order to make the simulator practical, it is important to improve its computation speed. In these simulations, it is essential to solve the Poisson equation and to calculate the associated Schrödinger eigenvalues $E_{\mathrm{n}}(\mathrm{x})$ every time step, in order to update the driving field according to the electron density. However, solving the Schrödinger equation every step costs a lot of computation time. That is why, in order to drastically accelerate the method, the Schrödinger equation is solved only every $N$ steps in our approach. Every other step, the eigenenergies $E_{\mathrm{n}}(\mathrm{x})$ are updated using a perturbative approach at the first order:

$$
\mathrm{E}_{\mathrm{n}}\left(\mathrm{x}_{0}\right)=\mathrm{E}_{\mathrm{n}}^{\mathrm{i}}\left(\mathrm{x}_{0}\right)+\left\langle\xi_{\mathrm{n}}^{\mathrm{i}}\left(\mathrm{x}_{0}, \mathrm{z}\right)\left|-\mathrm{q} \cdot \Delta \mathrm{V}\left(\mathrm{x}_{0}, \mathrm{z}\right)\right| \xi_{\mathrm{n}}^{\mathrm{i}}\left(\mathrm{x}_{0}, \mathrm{z}\right)\right\rangle
$$

where $\Delta V(x, z)$ refers to the difference between the updated electrostatic potential and that used for the last ' $i$ ' th Schrödinger solution. We found no need to correct the wavefunctions which are only updated every $\mathrm{N}$ steps.

It should be mentioned that we have finally chosen to solve the Schrödinger equation every $100^{\text {th }}$ time step. So, the part Schrödinger equation computation becomes negligible in the total computation-time compared to Poisson, scattering rates and transport calculations. If the frequency of the Schrödinger calculation is further more diminished, the expected reduction of the computation time will be small, and some instability may appear in the algorithm...

\section{RESULTS}

The simulated DGMOS has a gate length $L_{\mathrm{G}}$ equal to $L_{\mathrm{ch}}=15 \mathrm{~nm}$ (cf. Figure 1. ). The equivalent gate oxide thickness EOT and Si body $T_{\mathrm{Si}}$ thickness are equal to $1.2 \mathrm{~nm}$ and $3 \mathrm{~nm}$, respectively. The doping density is $N_{\mathrm{D}}=5 \times 10^{19} \mathrm{~cm}^{-}$ 3 in $\mathrm{N}^{+}$source-drain regions and the channel is undoped (residual doping: $N_{\mathrm{A}}=2 \times 10^{15} \mathrm{~cm}^{-3}$ ). The work function of the gate material is $4.46 \mathrm{eV}$. The supply voltage $V_{\mathrm{DD}}$ is $0.7 \mathrm{~V}$.

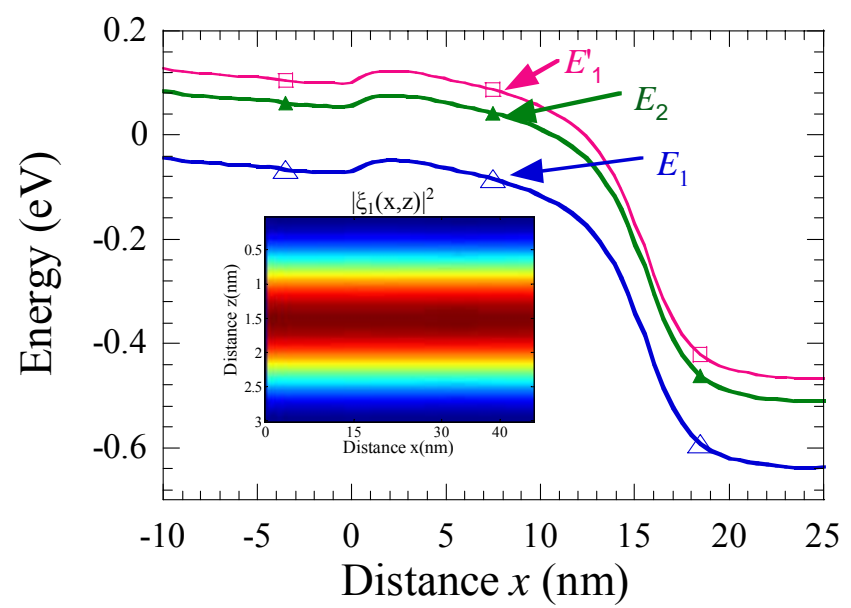

Figure 3. Quantized energy evolutions along $x$ axis. Inset: 2D cartography of square wave-function of the first sub-band. Unprimed and primed sub-bands have a quantization mass of $0.916 \mathrm{~m} 0$ and $0.19 \mathrm{~m} 0$, respectively

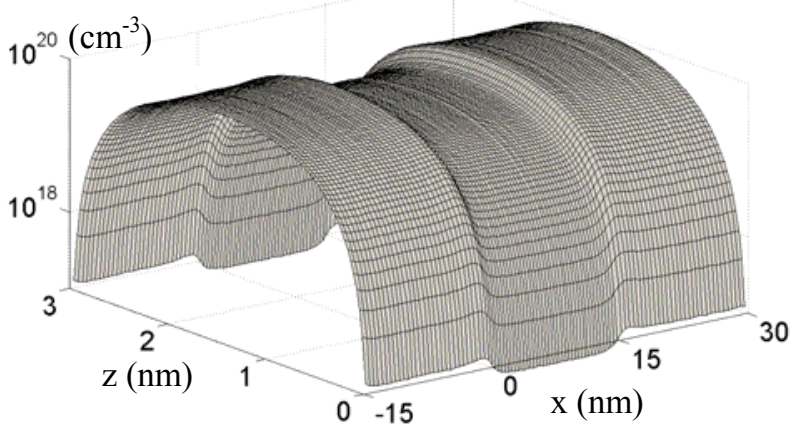

Figure 4. 2D cartography of electron density at $\mathrm{V}_{\mathrm{GS}}=0.7 \mathrm{~V}$ and $\mathrm{V}_{\mathrm{DS}}=0.1 \mathrm{~V}$.

In this work, we consider the 6 ellipsoidal $\Delta$ valleys of silicon conduction band. The "primed" valleys correspond to the valleys which have a transverse mass $m_{\mathrm{t}}$ along the confinement direction. The "unprimed" valleys refer to the valleys with a longitudinal mass $m_{1}$ along the confinement direction. The self-consistent sub-band profile obtained for $V_{\mathrm{GS}}=V_{\mathrm{DS}}=V_{\mathrm{DD}}=0.7 \mathrm{~V}$ is shown in Figure 3. As expected, the heaviest valleys in the confinement direction have the lowest quantized energies and the first primed sub-band has a higher energy: $E_{1}{ }^{\prime}>E_{2}>E_{1}$. Nevertheless, first primed subbands $\left(E_{1} '\right)$ are very close to the second unprimed sub-bands $\left(E_{2}\right)$ all along the device. It should be noted that the modespace approach is a very good approximation for this ultra-thin double gate structure symmetrically biased. Indeed, the coupling potentials between sub-bands, calculated as in Ref. [9], are negligible for this geometry since the wave functions 
do not evolve strongly along the source-drain axis, as illustrated in the inset of Figure 3. At $V_{\mathrm{GS}}=V_{\mathrm{DS}}=V_{\mathrm{DD}}=0.7 \mathrm{~V}$, i.e. for the most critical case, the perturbative coupling potentials remain smaller than $0.5 \mathrm{meV}$ which is lower than all energies involved (not shown).

Figure 4. indicates clearly that electrons are moved away from the $\mathrm{Si} / \mathrm{SiO}_{2}$ interface due to quantum repulsion in the whole structure, even in the source and drain regions.

The use of a perturbative solution of the Schrödinger equation makes the method drastically faster than in our previous work [6]. For results discussed below, equilibrium is obtained 15 times faster when solving Schrödinger's equation every 100 steps than when solving it every time step. Convergence is obtained without any loss of accuracy and no detectable difference in the results is seen. It should be noted that semi-classical simulation is still 3 times faster than the accelerated multi sub-band approach.

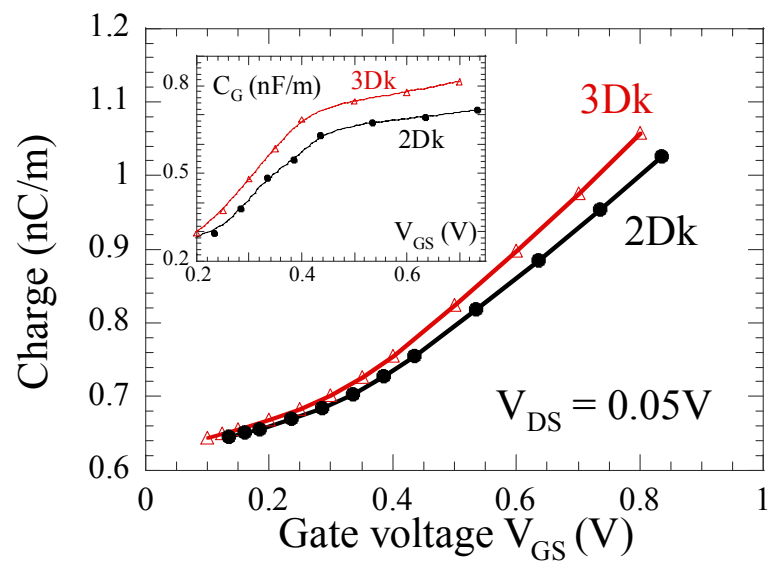

Figure 5. Charge vs. gate voltage characteristic at low $V_{\mathrm{DS}}$ obtained using semi classical MC simulation (triangles: 3Dk) and multi sub-band MC simulation (circles: 2Dk). Inset: Gate capacitance vs. gate voltage at low $V_{\mathrm{DS}}$

Combined with a particle multiplication technique to enhance the statistics of rare events, this improved multi-subband simulation approach makes possible the investigation of sub-threshold regime in a reasonable calculation time, which is known to be difficult in any particle Monte Carlo simulation. This improvement allows us to study the evolution of the total charge in the device and the gate capacitance as a function of the gate voltage as shown in Figure 5. and in the corresponding inset.

The gate control is softly degraded by the confinement effects in this $3 \mathrm{~nm}$ thick device. Indeed, the gate capacitance is reduced compared to classical simulation, which results from the dark space at $\mathrm{Si} / \mathrm{SiO}_{2}$ interfaces pointed out in Figure 4.

The electrical characteristics at low drain voltage are presented in Figure 6 . The confinement effects induce a $51 \mathrm{mV}$ increase of the threshold voltage $\mathrm{V}_{\mathrm{T}}$. In spite of the decrease of the gate capacitance observed previously, the sub-threshold slope remains excellent about $63 \mathrm{mV} / \mathrm{dec}$. in both $2 \mathrm{Dk}$ and 3Dk simulations. Indeed in this well designed device with a high enough form factor $\mathrm{L}_{\mathrm{ch}} / \mathrm{T}_{\mathrm{Si}}$ equal to 5 , short channel effects are successfully controlled. The $\mathrm{I}_{\mathrm{D}}-\mathrm{V}_{\mathrm{GS}}$ characteristics in the above threshold are shown in Figure 7. The predominant effect which limits the current for $\mathrm{V}_{\mathrm{GS}}$ higher than $0.6 \mathrm{~V}$ appears to be the total series resistances (about $170 \mu \Omega$.m).

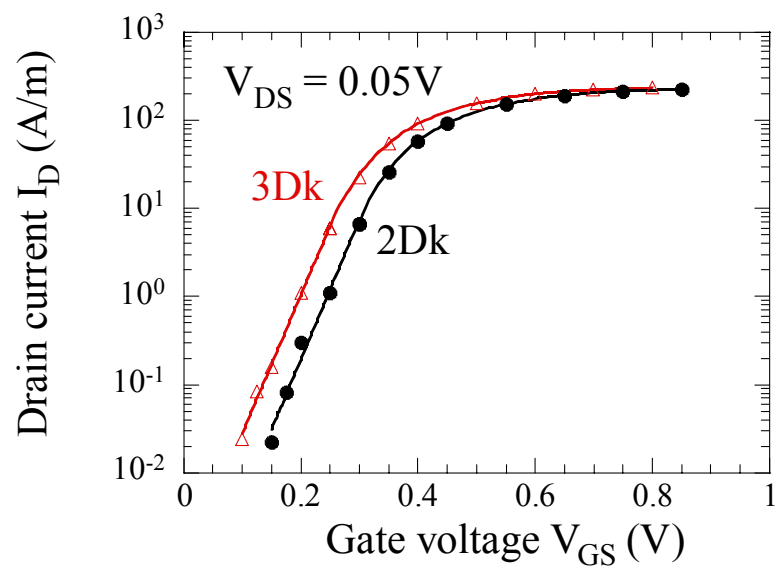

Figure 6. $I_{\mathrm{D}}\left(V_{\mathrm{GS}}\right)$ characteristic at low $V_{\mathrm{DS}}$ obtained using semi classical MC simulation (3Dk) and multi sub-band MC simulation (2Dk).

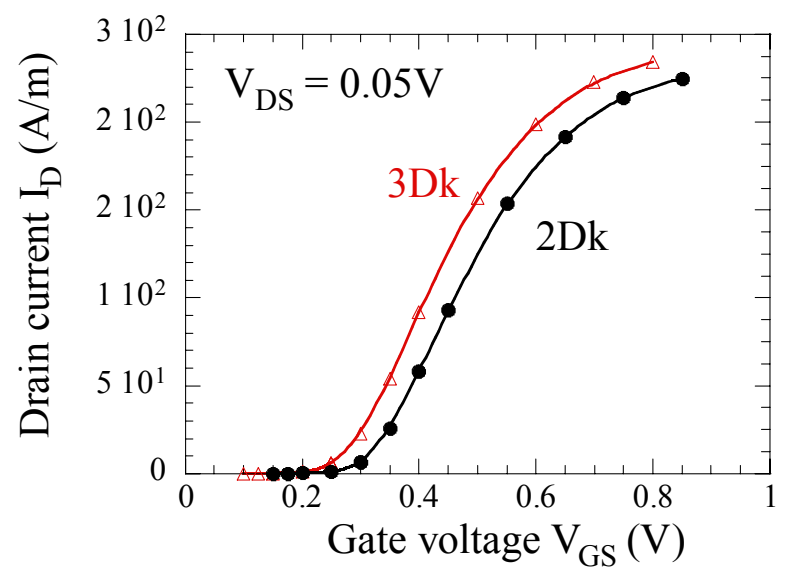

Figure 7. Charge vs. gate voltage characteristic at low $V_{\mathrm{DS}}$ obtained using semi classical MC simulation (3Dk) and multi sub-band MC simulation (2Dk).

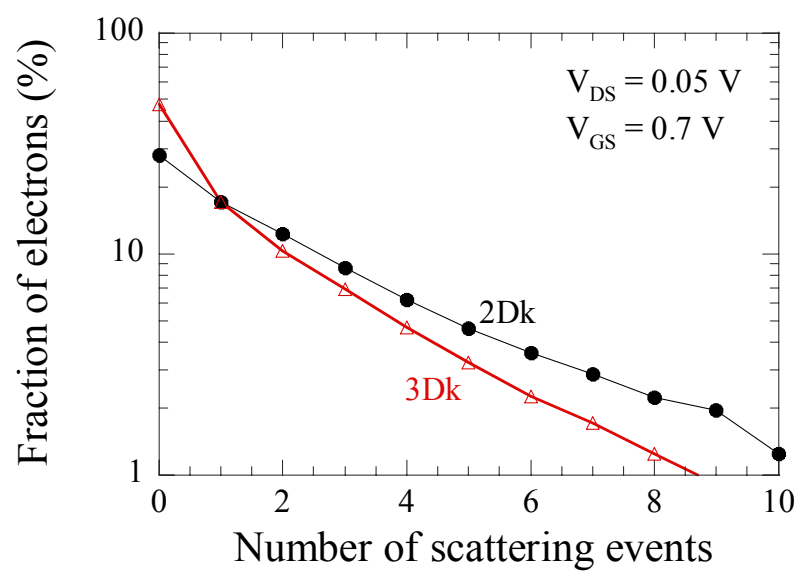

Figure 8. Fraction of electrons vs. the number of scattering events undergone at low $\mathrm{V}_{\mathrm{DS}}$ and high $\mathrm{V}_{\mathrm{GS}}$ during the channel crossing $(2 \mathrm{Dk}$ ). 
Finally, we have calculated the number of scattering events $N_{\text {scatt }}$ experienced by each carrier crossing the channel from source-end to drain-end. The resulting scattering spectra are plotted at low $\mathrm{V}_{\mathrm{DS}}$ and high $\mathrm{V}_{\mathrm{GS}}$ in Figure 8. as a function of $N_{\text {scatt }}$. Then, the fraction of ballistic electrons $B_{\text {int }}$ reaches $47 \%$ for the $3 \mathrm{Dk}$ simulation but decreases to $28 \%$ in the $2 \mathrm{Dk}$ case. Indeed, the roughness scattering (with $\Delta=0.5 \mathrm{~nm}$ and $\Lambda=1.5 \mathrm{~nm}$ ) induces a higher spreading of the spectrum in the 2Dk case because of high fluctuations of energy levels [7]. So, even if the ballisticity is high in this device the scattering mechanisms are far from being negligible.

\section{CONCLUSION AND PERPECTIVES}

We have developed a multi sub-band MC simulator for n-MOS transistors that extends the quantum confinement calculation to the whole device region. It also uses a simple and effective self-consistent iterative method that gives meaningful results with good convergence. This improved algorithm gives a more accurate description of density profile and carrier transport than semi-classical or quantum corrected $\mathrm{MC}$ simulation, with a reasonable increase of computation time. In particular, this approach makes possible the study of the subthreshold behavior of transistors.

Additionally, the accelerated multi sub-band approach make possible the coupling to a quantum 1D Wigner transport approach [10] in order to investigate the sub $10 \mathrm{~nm}$-long channel in which the quantum transport in the Source/Drain direction is predominant. This work is under development.

\section{REFERENCES}

[1] J. Saint-Martin, A. Bournel, and P. Dollfus, "Comparison of multiplegate MOSFET architectures using Monte Carlo simulation," Solid-State Electron., vol. 50, pp. 94-101, January 2006.
[2] J. Saint-Martin, A. Bournel, and P. Dollfus, "On the ballistic transport in nanometer-scaled DG MOSFET," IEEE Trans. Electron Dev. 51(7), 1148 (2004).

[3] R. Venugopal, Z. Ren, S. Datta, M. S. Lundstrom, and D. Jovanovic, "Simulating quantum transport in nanoscale MOSFETs: Real vs. mode space approaches," J. Appl. Phys., Vol. 92, pp. 3730-3739, October 2002.

[4] M. V. Fischetti and S. E. Laux, "Monte Carlo study of electron transport in silicon inversion layers," Phys. Rev. B, vol. 48, pp. 2244-2274, July 1993.

[5] F. Monsef, Philippe Dollfus, and Sylvie Galdin-Retailleau, "Electron transport in $\mathrm{Si} / \mathrm{SiGe}$ modulation-doped heterostructures using Monte Carlo simulation," J. Appl Phys. 95(7), 35870 (2004).

[6] S. M Goodnick, D. K. Ferry, C. W. Wilmsen, Z. Liliental, D. Fathy, and O. L. Krivanek, "Surface roughness at the $\mathrm{Si}(100)-\mathrm{SiO}_{2}$ interface," Phys. Rev. B 32(12), 8171 (1985).

[7] H. Sakaki et al., "Interface roughness scattering in GaAs/AlAs quantum wells," Appl. Phys. Lett. 51(23), 1934 (1987).

[8] J. Saint Martin, A. Bournel, F. Monsef, C. Chassat, and P. Dollfus, "Multi sub-band Monte Carlo simulation of ultra-thin Double Gate MOSFET with 2D electron gas," Semicond. Sci. Technol., Vol. 21, pp. L29-L31, April 2006.

[9] V. Sverdlov, A. Gehring, H. Kosina, and S. Selberherr, "Quantum transport in ultra-scaled double-gate MOSFETs: A Wigner functionbased Monte Carlo approach," Solid-State Electronics, 49, 1510 (2005).

[10] D. Querlioz, P. Dollfus, V. Nam Do, and V. Lien Nguyen, "An Improved Wigner Monte-Carlo Technique for the self-consistent Simulation of RTDs," in Proc. IWCE-11, 2006, to be published in J. Comput. Electron. 